\title{
Spatial homogeneity of the radiance of a large-diameter integrating sphere in the SWIR measured with an InGaAs camera
}

\author{
Sebastian König ${ }^{1}$, Berndt Gutschwager ${ }^{1}$, Ingmar Müller ${ }^{1}$, Richard D. Taubert ${ }^{1}$ \\ ${ }_{1}$ Physikalisch-Technische Bundesanstalt, Abbestr. 2-12, Berlin, Germany, \\ sebastian.koenig@ptb.de
}

\begin{abstract}
Summary:
We present the investigation of the spatial homogeneity of radiance of a large-diameter integrating sphere in the SWIR measured with an InGaAs camera. A procedure developed at PTB is used to correct the non-uniformity of the infrared camera used. Various configurations are examined and the suitability of the examined sphere for characterizing IR cameras. The integrating sphere was found having a homogeneity of $\pm 1 \%$ in the integral wavelength range from $0.9 \mu \mathrm{m}$ to $1.7 \mu \mathrm{m}$.
\end{abstract}

Keywords: Infrared camera, infrared, non-uniformity, metrological characterization

\begin{abstract}
Introduction
InGaAs (Indium Gallium Arsenide) detectors provide the highest specific detectivity $\left(D^{*}\right)$ of all detectors used in SWIR wavelength range at the lowest acquisition and operating costs [1]. This makes contactless temperature measurement with thermographic SWIR cameras available to a large number of users. For the characterization and calibration of infrared cameras, large-area radiation sources with a homogeneous radiation temperature distribution are required in order to be able to irradiate the entire field of view of the IR cameras homogeneously. For temperatures above $500{ }^{\circ} \mathrm{C}$, there are no large-area emitters available that would be capable of irradiate the entire field of view of an IR camera and have a good homogeneity of their radiation temperature over the entire area. Furthermore, when measuring in front of a largearea radiation source at high temperatures, the camera housing heats up. The effect of thermal load on cameras and methods for compensation have been investigated previously for cameras systems based on uncooled, shutterless microbolometer detector arrays [2]. Since InGaAs detector arrays differ fundamentally from thermal detectors with respect to the detection mechanism and spectral responsivity behavior, thermal loads and the associated, photon detector specific effects cannot be compensated applying methods for thermal detectors. Consequently, different approaches for characterization and calibration are required.
\end{abstract}

Hence, integrating spheres, especially when equipped with LEDs tailored for the spectral range of the device under test (DUT), are tuna- ble radiance sources without introducing a noticeable heat load on the DUT. However, the radiometric characterization of integrating spheres is cumbersome and time consuming, especially when the spatial dependence of the radiance is concerned [3]. In this work, the feasibility of a fast measurement method that can obtain the relative spatial uniformity of the radiance without being affected by the nonuniformity of the responsivity of the applied SWIR camera, the so-called DRM [4], is investigated. The DRM was originally developed as a non-uniformity correction for LWIR cameras. Here, it is used in SWIR for the first time.

\section{Measurement setup}

The large-aperture variable-radiance source (LAVRAS) [3] is based on an integrating sphere with a diameter of $1.2 \mathrm{~m}$, the radiating area has a diameter of $400 \mathrm{~mm}$ (Figure 1). The inner surface of the sphere is coated with $\mathrm{BaSO}_{4}$ for high reflectivity. As optical radiation sources 64 reflector type, $50 \mathrm{~W}$ tungsten halogen lamps with aluminum-coated reflectors (type OSRAM 64607 EFM) are used. 2 lamps are each connected in parallel as a pair. All 32 pairs can be controlled individually, each lamp can be continuously dimmed by means of a mechanical shutter. 3 broadband-filtered Si-photodiode detectors are used as monitor detectors in the UV, VIS and SWIR wavelength range. A more comprehensive description can be found in [3], whereas the theory of integrating spheres is explained in detail in [5]. The camera used is a SWIR InGaAs camera, sensitive between $0.9 \mu \mathrm{m}$ and $1.7 \mu \mathrm{m}$. The camera can only provide integral information 


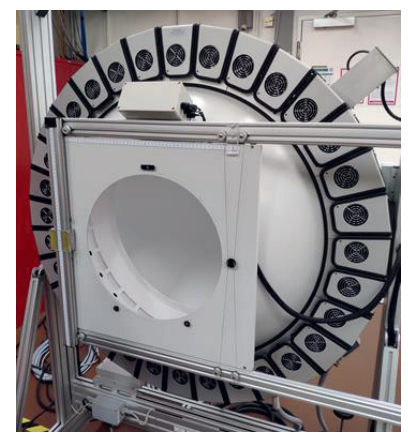

Fig. 1. The Large-Area, Variable Radiance Source (LAVRAS) of PTB

within its spectral range. However, by means of suitable optical filters, the spectral range can be narrowed and chosen as desired.

\section{Characterization of an integrating sphere}

The temporal stability of the sphere was monitored with the integrated detectors [3]. Sufficient stability over time is a prerequisite for using DRM. To determine the spatial homogeneity of the radiance from an integrating sphere, typically the aperture is scanned, with an imaging optical device e.g. with a radiation thermometer, which is very time-consuming. Therefore, the DRM is chosen to record the radiance proportional signals with a SWIR camera and to determine the spatial homogeneity of the radiance from this recording. However, a camera image is always a superposition of the recorded spatial radiation distribution and the non-uniformity of the spatial camera signal. By using the DRM, developed at PTB we are able to measure the spatial homogeneity of the radiance of the integrating sphere without being affected by the camera non-uniformity.

\section{Measurements}

The spatial homogeneity of the radiance of LAVRAS was measured for different settings. First, with the aid of the integrated lamp mechanical shutters, the radiance of LAVRAS was varied and the spatial homogeneity of the radiance was then examined. For this purpose, measurements were taken both with fully open shutters and with shutters half closed. In addition, the radiance homogeneity was examined when using all lamps and when using every second pair of lamps. In the third variation, the lights were turned off in the upper right quarter of the sphere.

\section{Results}

Figure 2 shows an example of the distribution of the radiance ratio based on a reference pixel in the center of the image. The lamp apertures of the integrating sphere were half-closed. The imaging geometry was chosen so that the corners of the field of view of the camera are just inside the integrating sphere aperture.

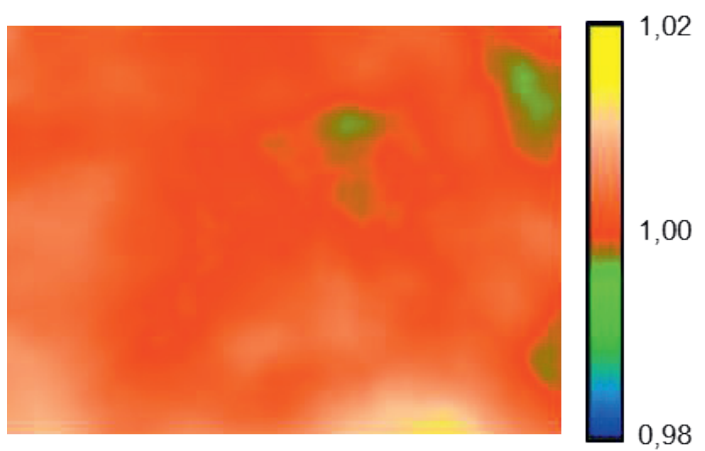

Fig. 2. Calculated distribution of the radiance ratio based on a reference pixel in the center of the integrating sphere, based on the measurement results with the InGaAs camera.

\section{Conclusion}

The integrating sphere was found having a homogeneity of $\pm 1 \%$ in the integral wavelength range from $0.9 \mu \mathrm{m}$ to $1.7 \mu \mathrm{m}$, measured with the DRM corrected InGaAs camera. This result is consistent with the results obtained in the UV up to the NIR [3]. If the radiance is reduced by closing the diaphragms on the lamps, it has been shown that the homogeneity of the radiation is only lightly affected. Similar results occur when the irradiation is varied by switching off different lamps. This indicates that the sphere coating is a close realization of a Lambertian reflector.

\section{Outlook}

In a next step, the measured radiance proportional values should be assigned to radiance temperatures. For this purpose, the SWIR camera is spectrally matched by optical filters to radiation thermometers or filter radiometers that are used to obtain the radiance temperatures of LAVRAS.

\section{References}

[1] Marc P. Hansen, Douglas S. Malchow: Overview of SWIR detectors, cameras, and applications, Proc. SPIE 6939, 2008

[2] A. Tempelhahn, H. Budzier, V. Krause, G. Gerlach: Improving the shutter-less compensation method for TEC-less microbolometer-based infrared cameras, SPIE Defense + Security. Baltimore, 2015. Bellingham: SPIE, Vol. 9451, 2015

[3] R. D. Taubert, C. Baltruschat, S. Schiller, and J. Hollandt: Radiometric characterization of a largeaperture variable-radiance calibration source for remote sensing applications, NEWRAD 2014, 2526, 2014

[4] Gutschwager B. and Hollandt J.: Nonuniformity correction of imaging systems with a spatially nonhomogeneous radiation source, Applied Optics 54, Issue 36, 10599-10605, 2015

[5] David G. Goebel: Generalized Integrating-Sphere Theory, Appl. Opt. 6, 125-128, 1967 